\title{
ORIGINAL RESEARCH \\ Isolated Progressive Visual Loss after Coiling of Paraclinoid Aneurysms
}

G.W. Schmidt

S.F. Oster

K.C. Golnik

L.M. Tumialán

V. Biousse

R. Turbin

C.J. Prestigiacomo

N.R. Miller
BACKGROUND AND PURPOSE: The proximity of the paraclinoid segment of the internal carotid artery to the visual pathways may result in visual deficits when patients present with aneurysms in this segment. Although surgical clip ligation of these aneurysms has been the standard of care for decades, the advent of coil embolization has permitted endovascular therapy in those aneurysms with favorable dome-to-neck ratios. Although immediate nonprogressive visual loss after coil embolization of paraclinoid aneurysms has been well described, isolated progressive visual loss immediately or shortly following coil embolization, to our knowledge, has not. We have identified 8 patients who experienced progressive loss of vision, unassociated with any other neurologic deficits, developing immediately or shortly after apparently uncomplicated coil embolization of a paraclinoid aneurysm.

MATERIALS AND METHODS: This study is a retrospective case series of 8 patients seen at 4 separate academic institutions. Inpatient and outpatient records were examined to determine patient demographics, previous ocular and medical history, and ophthalmic status before endovascular embolization. In addition, details of the primary endovascular therapy and subsequent surgical and nonsurgical interventions were recorded. Follow-up data, including most recent best-corrected visual acuity, postoperative course, and duration of follow-up were documented.

RESULTS: Eight patients developed progressive visual loss in 1 or both eyes immediately or shortly after apparently uncomplicated coiling of a paraclinoid aneurysm. MR imaging findings suggested that the visual loss was most likely caused by perianeurysmal inflammation related to the coils used to embolize the aneurysm, enlargement or persistence of the aneurysm despite coiling, or a combination of these mechanisms. Most patients experienced improvement in vision, 2 apparently related to treatment with systemic corticosteroids.

CONCLUSION: Patients in whom endovascular treatment of a paraclinoid aneurysm is contemplated should be warned about the potential for both isolated nonprogressive and progressive visual loss in 1 or both eyes. Patients in whom progressive visual loss occurs may benefit from treatment with systemic corticosteroids.
A neurysms of the internal carotid artery (ICA) that arise near the origin of the ophthalmic artery, sometimes called paraclinoid aneurysms, often present with neuro-ophthalmic signs and symptoms, such as visual loss from compressive or ischemic optic neuropathy and diplopia from ocular motor nerve compression or ischemia related to the proximity of these aneurysms to the afferent and efferent visual pathways. ${ }^{1}$ It is not surprising that direct surgical therapy of these aneurysms can be associated with substantial visual morbidity, particularly permanent loss of vision. ${ }^{2}$ It is for these reasons that alternative methods of treatment, particularly endovascular procedures, increasingly have been advocated.

During the past decade, endovascular placement of platinum coils into the sac of an unruptured aneurysm has become an accepted therapy for prevention or treatment of aneurysm rupture. ${ }^{3-5}$ The goal of this technique is to fill the sac with sufficient coils to induce thrombosis with partial collapse of the sac without occluding the parent vessel. The mechanism

Received December 25, 2006; accepted after revision April 23, 2007

From the Neuro-Ophthalmology Unit (G.W.S., S.F.O., N.R.M.), The Wilmer Ophthalmological Institute, Johns Hopkins University School of Medicine, Baltimore, Md; the Department of Neuro-Ophthalmology (K.C.G.), Cincinnati Eye Institute, University of Cincinnati, Cincinnati, Ohio; the Departments of Neurosurgery (L.M.T.), Neurology (V.B.), and Ophthalmology (V.B.), Emory University School of Medicine, Atlanta, Ga; and the Departments of NeuroOphthalmology and Orbital Surgery (R.T.) and Neurological Surgery and Radiology (C.J.P.), New Jersey Medical School, Newark, NJ.

Please address correspondence to Neil R. Miller, MD, Wilmer Ophthalmological Institute, Johns Hopkins Hospital, Maumenee 127, 600 N Wolfe St, Baltimore, MD 21287; e-mail: nrmiller@jhmi.edu

DOI 10.3174/ajnr.A0690 by which the coils induce thrombosis is incompletely understood but appears to be related to a cascade of events that include hemodynamic changes, thrombus formation, and fibrocellular proliferation.

The most frequent neurologic complication of coil embolization for intracranial aneurysm is thromboembolic stroke. ${ }^{6}$ Thrombus may form at the aneurysm neck on the surface of the coils or be flushed out of the coil mass by persistent flow, producing an embolus. Although strokes that occur in this setting may be characterized in part by loss of vision from retinal or optic nerve ischemia or the development of a homonymous visual field defect, such complications generally occur during or immediately following the procedure and usually are nonprogressive. In contrast, isolated progressive visual loss following endovascular coil embolization has rarely been reported. ${ }^{7}$ We describe 8 patients who experienced progressive loss of vision unassociated with any additional neurologic deficits immediately or shortly after apparently uncomplicated coiling of a paraclinoid aneurysm, and we discuss possible mechanisms for this phenomenon.

\section{Methods and Materials}

This study is a retrospective case series of 8 patients seen at 4 separate academic institutions. Inpatient and outpatient records were examined to determine patient demographics, previous ocular and medical history, and ophthalmic status before endovascular embolization. In addition, details of the primary endovascular therapy and subsequent surgical and nonsurgical interventions were recorded. Follow-up 


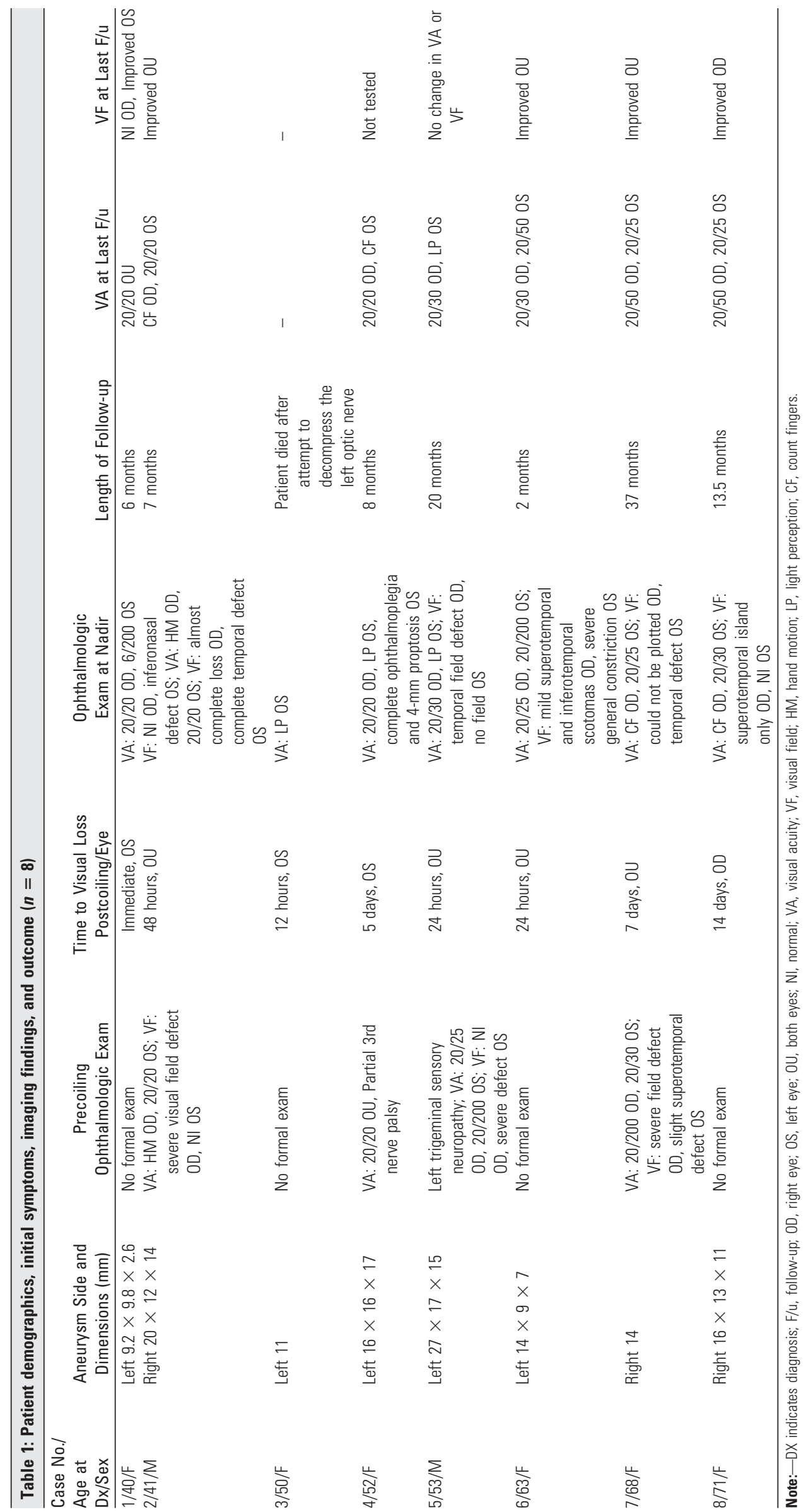




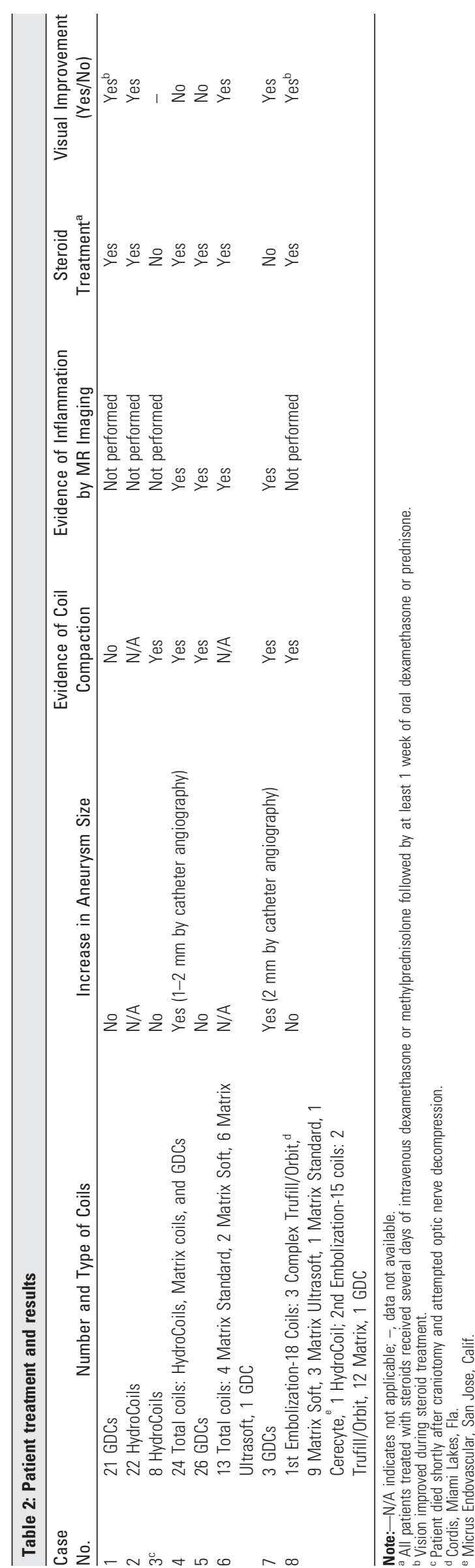

data, including most recent best-corrected visual acuity, postoperative course, and duration of follow-up were documented.

An attending neuro-ophthalmologist performed all ophthalmic examinations. When possible, testing of visual acuity was performed at a distance by using a Snellen acuity chart (Richmond Products, Albuquerque, NM) or close-up by using a Rosenbaum card (Richmond Products) with the patient's corrective lenses. If visual acuity improved with pinhole, this was recorded as the visual acuity at initial examination. If visual acuity was worse than $20 / 400$, it was designated as count fingers (CF) vision, detection of hand motion (HM), light perception (LP), or no light perception (NLP). The presence of a relative afferent pupillary defect (RAPD) was determined by using the swinging flashlight test. Visual field testing was performed by using a variety of techniques including kinetic perimetry, automated static perimetry, and confrontation, depending on the patient's visual acuity and clinical status. The appearance of the ocular fundus was assessed by using direct ophthalmoscopy, indirect ophthalmoscopy, or both.

\section{Results}

\section{Patient Population and Clinical Outcome}

Six patients were women and 2 were men; the mean age was 55 years (Table 1). The mean time to first symptoms of visual loss after coiling was 3.8 days, ranging from immediately after the procedure in 1 case (case 1) to 2 weeks after the procedure in another (case 8). All 8 patients experienced a further worsening of vision loss from the time of first symptoms, with a mean time interval to maximum vision loss of 27 days after coiling. Six of the 8 patients (cases 1, 2, 4, and 6-8) eventually experienced improvement in visual function after reaching their nadir; however, 1 patient (case 5) showed no change from nadir, and 1 patient (case 3) died shortly after an attempt was made to surgically decompress her optic nerve. No patient's vision returned to baseline during the period of follow-up, the mean length of which was 12 months.

\section{Neuroimaging and Posttreatment Characteristics}

Cases 1, 5, and 7 were treated with Guglielmi detachable coils (GDCs; Boston Scientific/Target, Fremont, Calif); cases 2 and 3, with HydroCoils (HydroCoil Embolic System; MicroVention, Aliso Viejo, Calif); and cases 4, 6, and 8, with a combination of HydroCoils, Matrix coils (Boston Scientific), and GDCs. Case 8 was the only one who underwent repeat endovascular coiling 8 weeks following initial coiling (Table 2). Following coiling, 2 patients (cases 4 and 7) showed an increase in overall aneurysm dimensions by conventional angiography, whereas in 4 patients (cases 1, 3, 5, and 8), the dimensions of the aneurysm were unchanged. Data regarding aneurysm size following coiling were not available in 2 cases (cases 2 and 6). Five of the 8 patients (cases $3-5,7$, and 8) showed evidence of coil compaction, 2 of whom (cases 4 and 7) were the ones in whom the aneurysm dimensions increased after coiling. MR imaging was performed at the time of visual loss in 4 of the 8 patients (cases $4-7$ ). In all 4 patients, the MR imaging was interpreted as showing evidence of perianeurysmal inflammation based on the presence of surrounding tissue that was hyperintense on $\mathrm{T} 2$-weighted images and that enhanced after intravenous injection of gadolinium diethylene triamine pentaacetic acid. Six of the 8 patients (cases 1, 2, 4-6, and 8 ) were treated with systemic corticosteroids, initially intravenously and then with oral agents. Two patients were not treated with steroids. 

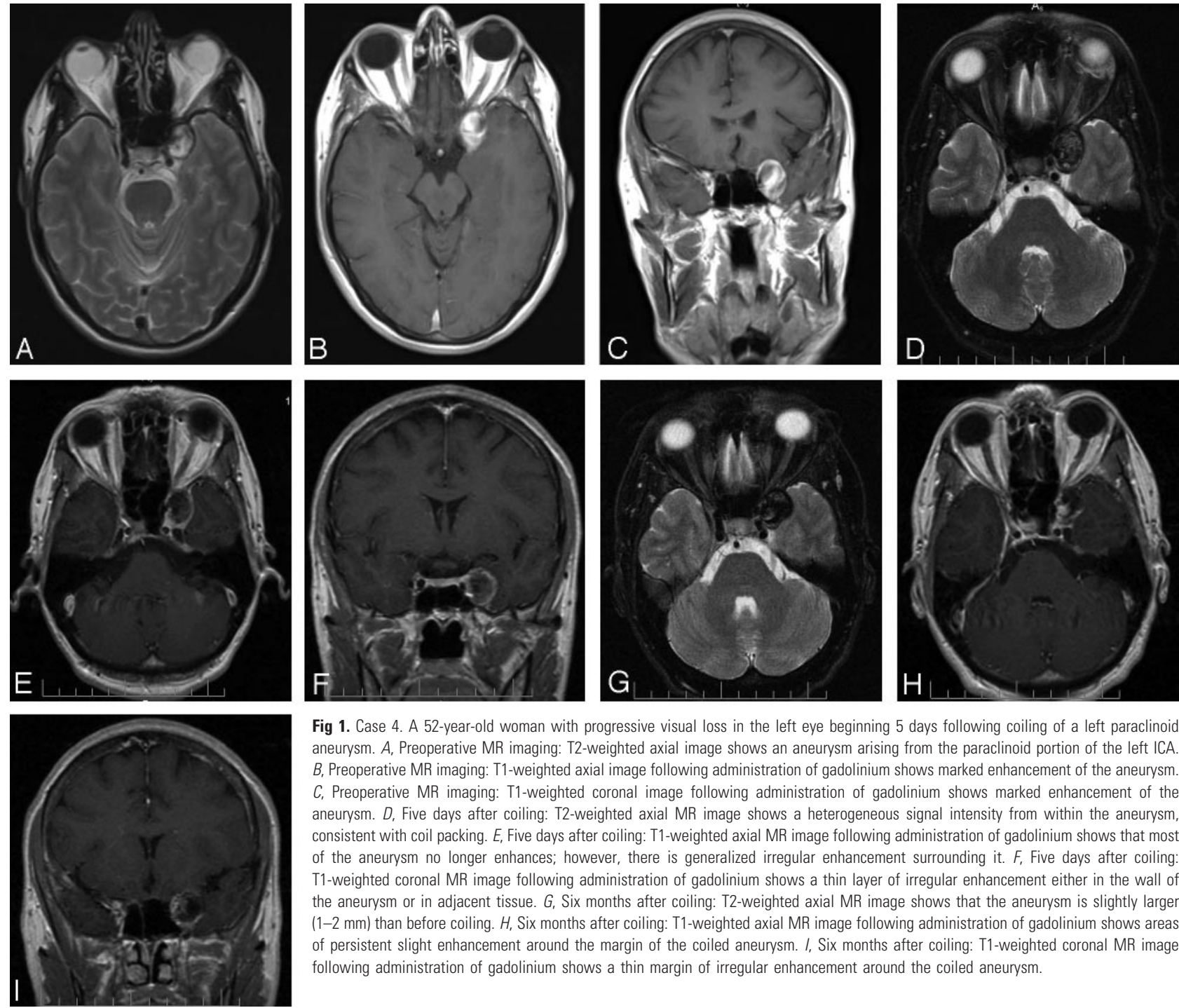

Fig 1. Case 4. A 52-year-old woman with progressive visual loss in the left eye beginning 5 days following coiling of a left paraclinoid aneurysm. A, Preoperative MR imaging: T2-weighted axial image shows an aneurysm arising from the paraclinoid portion of the left ICA. $B$, Preoperative MR imaging: T1-weighted axial image following administration of gadolinium shows marked enhancement of the aneurysm. C. Preoperative MR imaging: T1-weighted coronal image following administration of gadolinium shows marked enhancement of the aneurysm. D, Five days after coiling: T2-weighted axial MR image shows a heterogeneous signal intensity from within the aneurysm, consistent with coil packing. E, Five days after coiling: T1-weighted axial MR image following administration of gadolinium shows that most of the aneurysm no longer enhances; however, there is generalized irregular enhancement surrounding it. $F$, Five days after coiling: T1-weighted coronal MR image following administration of gadolinium shows a thin layer of irregular enhancement either in the wall of the aneurysm or in adjacent tissue. G. Six months after coiling: T2-weighted axial MR image shows that the aneurysm is slightly larger $(1-2 \mathrm{~mm})$ than before coiling. $H$, Six months after coiling: T1-weighted axial MR image following administration of gadolinium shows areas of persistent slight enhancement around the margin of the coiled aneurysm. I, Six months after coiling: T1-weighted coronal MR image following administration of gadolinium shows a thin margin of irregular enhancement around the coiled aneurysm.

One was the patient who died shortly after a craniotomy to decompress the optic nerve (case 3).

\section{Representative Cases}

Case 1. A 40-year-old woman underwent coiling of 2 left paraclinoid aneurysms. Twenty-one GDCs were placed sequentially into the largest aneurysm, which measured $9.2 \times 9.8 \times 2.6 \mathrm{~mm}$. Two coils were then placed sequentially into the second smaller paraclinoid aneurysm.

Immediately after the procedure, the patient began to experience deterioration of vision in her left eye. Eight days after surgery, she was evaluated and found to have a left retrobulbar optic neuropathy characterized by visual acuity of 20/50, decreased color vision, an inferior visual field defect, and a left RAPD. The patient was treated with intravenous dexamethasone and underwent CT angiography, which revealed no change in the size or shape of the coil mass. After 2 days of treatment with intravenous dexamethasone, the patient was discharged on oral dexamethasone. During the next week, her vision decreased further to 6/200; however, it then began to improve, such that 1 month after coiling, visual acuity in the eye was 20/200, and 6 months after coiling, visual acuity was $20 / 20$ in the eye, with normal color perception and a minimal residual inferior nasal defect in the visual field.
Despite lack of neuroimaging confirmation, the patient's improvement within approximately 1 week of being placed on corticosteroids suggests that the visual loss in this case was caused by perianeurysmal inflammation that responded, at least in part, to treatment with systemic corticosteroids. We cannot, however, exclude the possibility that postcoiling aneurysmal mass effect also played a role.

Case 4. A 52-year-old woman noticed a gradual decline of visual acuity in her left eye. She was subsequently found to have visual acuity of 20/20 in both eyes, but there was a mild left RAPD. The patient also had a paresis of the superior division of the left third nerve and a left trigeminal sensory neuropathy. MR imaging revealed a paraclinoid aneurysm arising from the left ICA (Fig $1 A-C)$, and an angiogram revealed a partially thrombosed aneurysm measuring $16 \times 16 \times 17 \mathrm{~mm}$. Endovascular embolization of the aneurysm was performed by using 24 coils, including a combination of GDCs, HydroCoils, and Matrix coils. Initially, the patient had no postoperative visual symptoms; however, 5 days following coiling, she noted decreased vision in the left eye. MR imaging and MR angiography showed a $24 \times 17 \times 20 \mathrm{~mm}$ mass lateral to the cavernous portion of the left ICA, with a heterogeneous signal intensity that was hypointense in some areas and hyperintense in others on T2-weighted images, consistent 

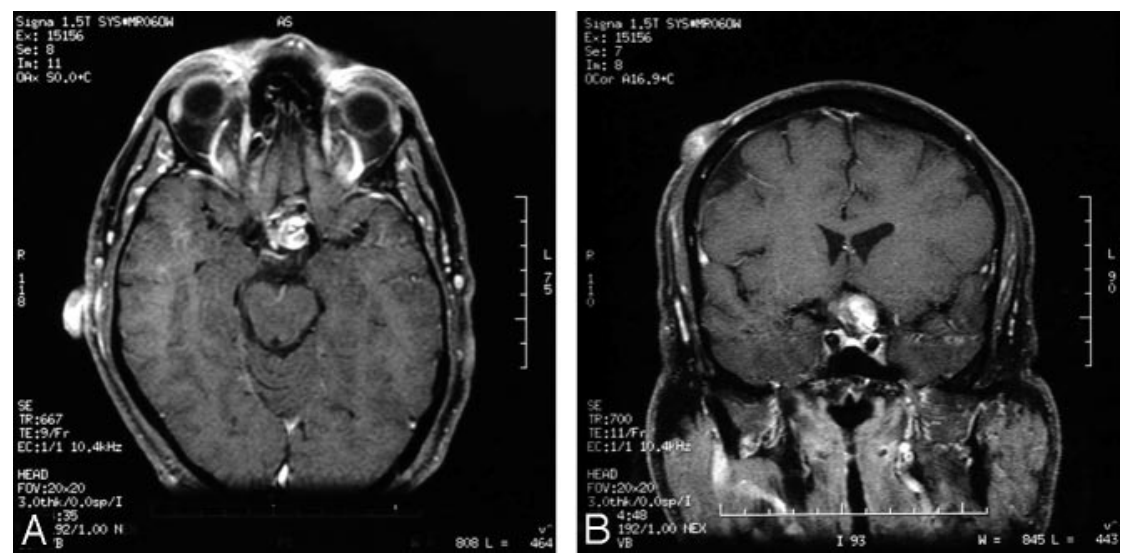

with a partially thrombosed aneurysm (Fig 1D). Following administration of gadolinium, there was peripheral enhancement surrounding the mass, consistent with perianeurysmal inflammation (Fig $1 E,-F$ ). The patient was treated with intravenous methylprednisolone, $250 \mathrm{mg}$ every 6 hours for 3 days, and then was maintained on oral prednisone, $60 \mathrm{mg}$ daily for 2 weeks. During this time, her acuity remained normal in the right eye but decreased to LP in the left eye, associated with a left RAPD. She also developed a complete left ophthalmoplegia, $4 \mathrm{~mm}$ of left proptosis, and hypesthesia in the distribution of the left trigeminal nerve. Given the lack of a significant response to steroids, prednisone was tapered following 2.5 weeks of treatment. The patient was followed at regular intervals, and 6 months after embolization, neuroimaging showed compaction of the coiling material with recanalization of the aneurysm, which was now 1-2 mm larger than before coiling (Fig $1 G$ ). There was still enhancement around the margin of the mass (Fig $1 H,-I$ ). Given the coil compaction, the option of repeat embolization was discussed with the patient, but she declined further intervention. Eight months after embolization, her visual acuity was stable at 20/20 in the right eye and CF at 2 feet in the left eye. Her ocular motility had improved, and she now had a persistent left oculomotor nerve paresis with evidence of aberrant regeneration.

We believe the MR imaging findings were consistent with perianeurysmal inflammation and that the visual loss in this patient was caused by a combination of inflammation and postcoiling enlargement of the partially occluded aneurysm. The patient's lack of response to corticosteroid treatment suggests that the latter mechanism was the more significant and may have prevented further recovery of vision.

Case 5. A 53-year-old man had progressive decreased vision in the left eye. An evaluation revealed that the visual acuity and visual field in the right eye were normal; however, there was a left retrobulbar optic neuropathy characterized by visual acuity of 20/200, a significant visual field defect, a RAPD, and a normalappearing optic disc. MR imaging showed changes consistent with a large paraclinoid aneurysm originating from the left ICA, confirmed by subsequent catheter angiography, which showed that it measured $27 \times 17 \times 15 \mathrm{~mm}$. About 24 hours after endovascular coiling with 26 GDCs, the patient developed a severe headache and sudden worsening of vision in the left eye. MR imaging revealed a large heterogeneous mass in the suprasellar region that partially enhanced following gadolinium administration. There was also slight enhancement of surrounding tissue (Fig 2). Conventional angiography showed continued flow into the aneurysm with compaction of the coils. The patient was
Fig 2. Case 5. A 53-year-old man with progressive visual loss in the left eye beginning 24 hours following coiling of a left paraclinoid aneurysm. T1-weighted axial $(A)$ and coronal $(B)$ MR images show irregular enhancement, suggesting recanalization of the aneurysm as well as mildly diminished flow within the left cavernous and supraclinoid ICA, thought to be related to mass effect from the partially occluded aneurysm.

treated with a short course of intravenous dexamathasone, followed by oral dexamethasone. During the next month, despite continued steroid treatment, the visual acuity in the left eye decreased to NLP. Although the visual acuity in the right eye remained normal, perimetry demonstrated a new temporal visual field defect. No further treatment was recommended, and the patient's vision remained stable during the subsequent 20 months.

We believe this patient's visual loss was related mainly to mass effect from the aneurysm that was incompletely occluded by the coils. This may explain why the patient's visual function worsened despite treatment with systemic corticosteroids.

Case 8. A 71-year-old woman underwent MR imaging because of dizziness. The MR imaging revealed changes consistent with a right paraclinoid aneurysm. Diagnostic angiography confirmed a multilobed $16 \times 13 \times 11 \mathrm{~mm}$ right paraclinoid-ophthalmic artery aneurysm (with a 9.2-mm neck) extending along the superior wall of the supraclinoid portion of the ICA. The patient elected to undergo embolization of the aneurysm. Before coiling, the patient underwent visual field testing. The visual field of the right eye showed mild superior and inferior depression. The visual field of left eye was normal. Endovascular embolization was subsequently performed by using 18 coils of various types. Immediately after the procedure, findings of the patient's neurologic examination were normal and her visual function was stable. Two weeks after embolization, however, she awoke with painless visual loss in the right eye. Examination revealed visual acuity of CF in that eye, associated with a worse visual field defect, and a right RAPD. Left eye visual function was normal. Cerebral angiography demonstrated compaction of coils at the anterior portion of the dome of the aneurysm with persistent flow within the aneurysm (Fig $3 A$ ). The patient was treated with intravenous methylprednisolone, $500 \mathrm{mg}$ per day for 3 days, followed by 60 mg of prednisone per day, tapered during 3 weeks. Within 3 weeks after embolization, the patient's visual acuity had improved to 20/200 in the right eye and visual field testing revealed improvement in the visual field. Six weeks after embolization, neuroimaging demonstrated that the aneurysm was only partially occluded by the coils. Eight weeks after coiling, the patient's vision had improved to 20/60 in the right eye and was 20/25 in the left eye, with significant improvement in the right eye visual field (Fig 3B).

Because of persistent flow in the aneurysm, the patient was treated with further coiling. Fifteen additional coils were deployed in the anterior and posterior compartments of the aneurysm. No changes in vision were noted in the immediate postcoiling period. The patient was examined at regular intervals, and at her last evaluation, 13.5 months after the initial coiling, the visual acuity in the right eye was stable but there was further improvement in the visual field (Fig $3 C-E$ ).

The pathogenesis of visual loss in this patient is unclear. A 


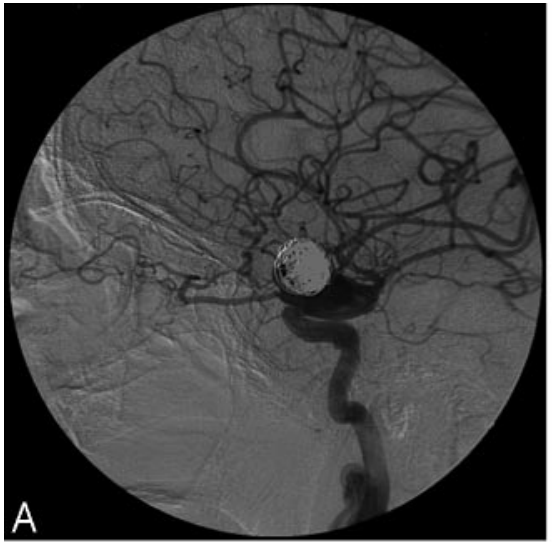

Fig 3. Case 8. A 71-year-old woman with progressive visual loss in the right eye beginning 2 weeks following coiling of a right paraclinoid aneurysm. $A$, Conventional angiogram following endovascular embolization demonstrates compaction of coils at the anterior portion of the aneurysm. $B$, Automated static perimetry 8 weeks postcoiling reveals a field defect in the right eye and a minimal superior temporal defect in the left eye. $C$, Eleven weeks after coiling, the visual field (automated static perimetry) of the right eye

shows minimal worsening and there is now a more significant superior temporal defect in the visual field of the left eye. $D$, Thirty-nine weeks after coiling, the visual field (automated static perimetry) of the right eye shows improvement and there is minimal change in the superior temporal defect in the visual field of the left eye. $E$, Automated static perimetry 54 weeks after the coiling procedure reveals an improved superior and inferior arcuate scotoma on the right side. The left eye reveals a subtle superotemporal defect.

water-hammer effect from the partially occluded aneurysm may have been responsible; however, the improvement of visual function during treatment with systemic steroids suggests that perianeurysmal inflammation may have played a role.

\section{Discussion}

Traditionally, surgical clipping has been the treatment of choice for most intracranial aneurysms; however, paraclinoid aneurysms present a particular challenge because their anatomy and proximity to the anterior clinoid processes and relationship to the dural ring may preclude such treatment. ${ }^{8}$ Until recently, many of these aneurysms were wrapped with cotton gauze, often reinforced with fibrin glue or a similar substance, or left untreated. Although in many cases, an aneurysm can be successfully stabilized by wrapping, in some, an excessive inflammatory reaction develops that may cause loss of vision that may or may not respond to treatment with systemic corticosteroids. ${ }^{9,10}$ Thus, many authors now warn against the use of cotton wrapping near the optic nerves and chiasm. ${ }^{11}$

Advances in microcatheter technology, endovascular techniques, and embolization materials have increased the popularity of neurointerventional therapy for paraclinoid aneurysms. ${ }^{4}$ The appeal of endovascular occlusion of cerebral aneurysms is that it is a less invasive technique and has been shown to be generally safer than clipping. Johnston et $\mathrm{al}^{5}$ reported on 2069 patients treated for unruptured intracranial aneurysms between 1990 and 1998 and quoted a mortality rate of $3.5 \%$ for clipping versus $0.5 \%$ for coiling. Additionally, a prospective, randomized, controlled trial, the International Subarachnoid Aneurysm Trial, found that patients who underwent endovascular coiling of ruptured intracranial aneurysms had a $6.9 \%$ absolute risk reduction in dependency or death at 1 year, compared with those who underwent surgical clipping. ${ }^{12}$ However, with regard to visual loss with coiling versus clipping in the treatment of paraclinoid aneurysms, Hoh et $\mathrm{al}^{13}$ reported no difference in complications.
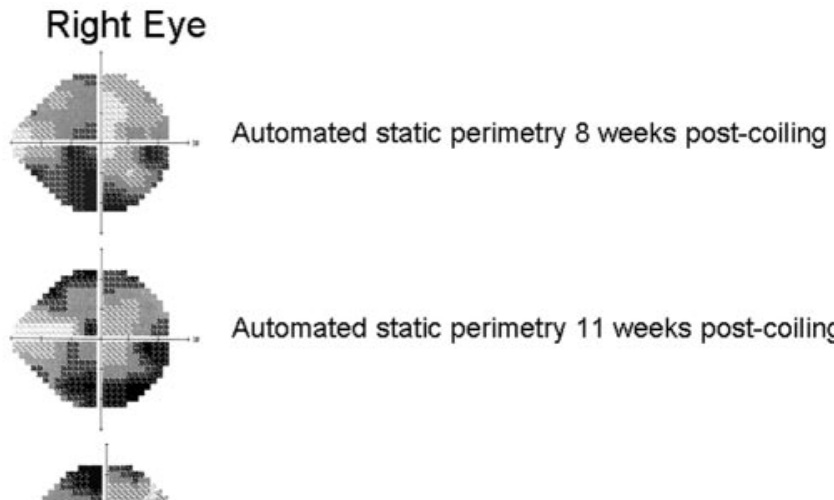

Automated static perimetry 11 weeks post-coiling

Automated static perimetry 39 weeks post-coiling

Automated static perimetry 54 weeks post-coiling

The most frequent neurologic complication of coil embolization for intracranial aneurysm is thrombo-embolic stroke. ${ }^{6}$ Strokes that occur in this setting may be associated with ipsilateral loss of vision from retrobulbar ischemic optic neuropathy, central retinal artery occlusion, or ocular ischemic syndrome. They may also be characterized in part by homonymous visual field defects. Our 8 patients experienced isolated progressive vision loss immediately (case 1) or shortly following (cases 2-8) endovascular coil embolization. This outcome has not been previously reported as a complication and is not consistent with the immediate postoperative vision loss usually associated with surgical trauma or acute ischemia. We believe the mechanism for postcoiling progressive visual loss is multifactorial, resulting from perianeurysmal inflammation produced by the thrombogenic coils, from aneurysm growth because of failure to obliterate the aneurysm sac or from coil compaction with continued compression of the visual apparatus, or from a combination of both processes. Although ischemia probably is responsible for the immediate nonprogressive visual loss that has been reported in some patients following coil embolization of paraclinoid aneurysms, ${ }^{2}$ we believe that this mechanism is unlikely to be responsible for the progressive visual loss experienced by our 8 patients, particularly given the delay in visual symptoms by at least 24 hours in 7 of the 8 patients and the apparent response to steroid treatment in 4 of the 6 treated patients.

Very few human studies of the histologic and hemodynamic effects of coil embolization have been performed, but numerous animal models have been generated. Studies of the histologic effects of embolization with coils have been performed in rabbits, ${ }^{14-16}$ pigs,${ }^{17,}{ }^{18}$ dogs,${ }^{19-21}$ and nonhuman primates, ${ }^{22}$ providing clues to the pathogenesis of vision loss in our 8 human patients.

Evidence for an inflammatory process after coiling comes from the rabbit aneurysm model of Fujiwara and Kallmes, ${ }^{15}$ in which there was a time-dependent response to coil packing. At 2 
weeks, an unorganized clot with fibrin and inflammatory cells was present at the aneurysm neck. Böcher-Schwarz et $\mathrm{al}^{23}$ reported the histology in a rabbit carotid artery bifurcation aneurysm model. After coil embolization, the aneurysm wall became disorganized from invasion by capillaries and fibroblasts, organization of the thrombotic material, and the initiation of a chronic foreign body reaction in the neighborhood of the coils. Twelve weeks after application of platinum coils into the aneurysm sac, the aneurysm lumen was completely obliterated by cellular and vascularized granulation tissue consisting of fibrocytes, numerous vessels, and capillaries. There was severe chronic inflammation with numerous foreign body giant cells (FBGCs) at the interface between coils and tissue. Dai et $\mathrm{al}^{16}$ evaluated the cellular mechanisms of aneurysm healing with a longitudinal immunohistochemical study in a rabbit model. These investigators showed that early after coil implantation, the aneurysm dome primarily was composed of thrombus; however, within this thrombus, centripetal infiltration of myofibroblasts rapidly occurred, resulting in inflammation. In this study, the inflammation was confined to the lumen and did not involve the wall of the aneurysm.

These histopathologic results are consistent with the findings in the few anecdotal case reports and small case series that make up most of the available human studies. ${ }^{24-26}$ For example, Bavinzski et $\mathrm{al}^{26}$ examined 17 aneurysms in 15 patients who died after treatment with GDCs. A polymorphonuclear leukocytic infiltrate was present within the lumen near the aneurysm wall in those aneurysms that had been treated within 1 week of the patient's death. At 9 and 14 days after embolization, fibroblasts were noted to invade the wall. In aneurysms examined on days 22 and 40 postimplantation, granulation tissue and connective tissue proliferation and capillary ingrowth were present within the lumen adjacent to the aneurysm wall. In an aneurysm removed at surgery on the 54th month postprocedure, thick fibrous tissue, collagen, small blood vessels larger than capillaries, and even some of the coils themselves had invaded the aneurysm wall. In all human aneurysms studied, numerous inflammatory cells, especially FBGCs, were found close to the outer and inner surface of the coils.

It thus seems clear from both experimental animal models and human anatomic studies that packing of aneurysms with coils produces an inflammatory response characterized in part by numerous FBGCs at the outer and inner surface of platinum coils. The chronic inflammation may cause thickening of the aneurysm wall, making this an important protective mechanism to prevent aneurysm rupture. In fact, accelerating the process of thrombus organization and fibrosis has been considered a favorable characteristic for aneurysm exclusion. Ding et $\mathrm{al}^{27}$ showed in a rabbit model that the use of Matrix coils, which carry a biodegradable polymer designed to amplify tissue response to coils, increased the inflammatory reaction. Cases 4 and 6 in our series, who were treated with Matrix coils, are 2 of the 4 patients with neuroimaging evidence of an inflammatory process surrounding the paraclinoid coil mass. This response suggests that the enhanced biologic activity of Matrix coils, aimed at producing a fibrotic reaction, can increase the potential for inflammation-related complications such as optic nerve-related visual loss and other cranial neuropathies. In this same study, Ding et $\mathrm{al}^{27}$ showed that coil compaction occurred more frequently in the Matrix group than in the HydroCoil or platinum coil groups; however, a recent report by $\mathrm{Im}$ et $\mathrm{al}^{28}$ described 3 cases of aseptic meningitis following embolization of intracranial aneurysms with HydroCoils, indicating that both types can induce inflammatory changes.

Chronic inflammation may injure adjacent neural structures, including the afferent and efferent visual pathways, not only from the direct effects of the inflammation itself but also by several other mechanisms. For example, the small blood vessels can become occluded, resulting in ischemia. Alternatively, the inflammatory response can produce a mass effect. The postembolization MR imaging changes in cases $4-7$, a postembolization fever that occurred in cases 6 and 8 , and the subsequent improvement in vision in 5 of the 8 patients, 2 of whom (cases 1 and 6) were being treated with corticosteroids at the time of improvement, are consistent with this hypothesis. Thus, although 2 of our patients with presumed postcoiling inflammation did not respond to treatment with steroids and 4 improved after steroids were stopped, we believe that this therapy should still be considered in cases of isolated progressive vision loss after endovascular coiling of paraclinoid aneurysms, particularly when the aneurysm appears to have been successfully occluded and there is imaging evidence of inflammation. Indeed, Stracke et $\mathrm{al}^{29}$ recently reported the case of a patient similar to several of ours who experienced dramatic improvement in vision when treated with systemic corticosteroids after experiencing severe visual loss in the right eye 24 hours after treatment of a right supraophthalmic aneurysm with a framing GDC and 8 bioactive Matrix coils. MR imaging in this patient revealed marked thickening and enhancement of the optic chiasm adjacent to the coiled aneurysm, with severe edema extending into the right optic nerve and both optic tracts. All of these abnormalities completely regressed after about 1 week of therapy with dexamethasone.

In addition to perianeurysmal inflammation, aneurysm enlargement after incomplete coil embolization is another potential mechanism for injury to the visual pathways. Inadequate coil volume or a change in coil configuration may result in the persistence or re-establishment of blood flow within the interstices of the coils and a potential "ball-valve" mechanism, resulting in the continued growth or regrowth of the aneurysm after initial stabilization. ${ }^{30}$ Studies show that if the aneurysm is not tightly packed with coils and a residual neck is present, the constant pulsation of arterial blood flow from the parent vessel against the coils and into the residual neck - the "water-hammer effect"31_may cause the coils to change configuration, cluster together, and move toward the dome of the aneurysm. ${ }^{32-35}$

Fernandez Zubillaga et $\mathrm{al}^{34}$ showed that immediate and complete occlusion can be achieved in 85\% of small-necked aneurysms, but wide-necked aneurysms are less often densely and completely packed because of the risk of coils herniating into the parent vessel. Imperfect packing attenuation together with exposure of a large surface area to the water-hammer effect leads to incomplete occlusion in most wide-necked aneurysms (approximately $85 \%),{ }^{34}$ with subsequent progressive compaction of coils and the formation of aneurysmal remnants.

Viñuela et $\mathrm{al}^{36}$ reported a neck remnant in $21 \%$ of small aneurysms with small necks, $42 \%$ of small aneurysms with wide necks, $51 \%$ of large aneurysms, and $50 \%$ of giant aneurysms after endovascular coil embolization. Hayakawa et $\mathrm{al}^{37}$ reviewed their experience with the natural history of residual neck remnants after GDC treatment and after a mean follow-up of 17.3 months; 
$25 \%$ of residual aneurysm necks had angiographic evidence of complete thrombosis of the aneurysm, 26\% remained unchanged, and 49\% demonstrated recanalization of the aneurysm. Thornton et $\mathrm{al}^{38}$ found that $46 \%$ of aneurysms with postembolization residual necks had angiographic evidence of progressive thrombosis, 26\% remained stable, and 28\% demonstrated enlargement of the residual neck after a mean follow-up time of 16.7 months. Enlargement of postembolization residual necks may present with neuro-ophthalmic manifestations, as described by Bhatti et al, ${ }^{39}$ who reported a case of delayed exacerbation of a third nerve palsy due to aneurysmal regrowth. Because of their large size, nonspheric shape, and wide neck, paraclinoid aneurysms can be particularly difficult to pack and obliterate. We believe this mechanism may explain the aneurysm regrowth in cases 1,3 , and 4 .

\section{Conclusion}

We report 8 cases of isolated progressive vision loss after endovascular coiling of paraclinoid aneurysms. We believe this complication is the result of an inflammatory process induced by the coils in some cases, whereas in others, aneurysm enlargement following incomplete packing of the aneurysm or compaction of coils may be responsible. In still others, a combination of these mechanisms may act in conjunction with each other to cause the visual manifestations. Patients in whom endovascular coiling of a paraclinoid aneurysm is contemplated should be warned about the potential for both nonprogressive and progressive visual loss in 1 or both eyes. Fortunately, there appears to be a good chance of improvement that may or may not be related to steroid therapy. We, therefore, believe that patients in whom progressive visual loss occurs should be treated with systemic corticosteroids, particularly if the aneurysm is found to be completely occluded and there is evidence of inflammation by MR imaging because this may result in stabilization or improvement in visual function.

\section{References}

1. Kasner SE, Liu GT, Galetta SL. Neuro-ophthalmologic aspects of aneurysms. Neuroimaging Clin North Am 1997;7:679-92

2. Kattner KA, Bailes J, Fukushima T. Direct surgical management of large bulbous and giant aneurysms involving the paraclinoid segment of the internal carotid artery: report of 29 cases. Surg Neurol 1998;49:471-80

3. Lanterna LA, Tredici G, Dimitrov BD, et al. Treatment of unruptured cerebral aneurysms by embolization with Guglielmi detachable coils: case-fatality, morbidity, and effectiveness in preventing bleeding - a systematic review of the literature. Neurosurgery 2004;55:767-75

4. Brilstra EH, Rinkel GJ, van der Graaf Y, et al. Treatment of intracranial aneurysms by embolization with coils: a systematic review. Stroke 1999;30:470-76

5. Johnston SC, Zhao S, Dudley RA, et al. Treatment of unruptured cerebral aneurysms in California. Stroke 2001;32:597-605

6. Derdeyn CP, Cross DT 3rd, Moran CJ, et al. Postprocedure ischemic events after treatment of intracranial aneurysms with Guglielmi detachable coils. J Neurosurg 2002;96:837-43

7. Tawk RG, Villalobos HJ, Levy EI, et al. Surgical decompression and coil removal for the recovery of vision after coiling and proximal occlusion of a clinoidal segment aneurysm: technical case report. Neurosurgery 2006;58:1217-18

8. Fox JL. Microsurgical treatment of ventral (paraclinoid) internal carotid artery aneurysms. Neurosurgery 1988;22:32-39

9. Bhatti MT, Holder CA, Newman NJ, et al. MR characteristics of muslin-induced optic neuropathy: report of two cases and review of the literature. AJNR Am J Neuroradiol 2000;21:346-52

10. Subramanian PS, Miller NR, Renard V, et al. Delayed progressive visual loss following wrapping of bilateral clinoidal aneurysms: recovery of vision and improvement in neuroimaging during corticosteroid treatment. BrJOphthalmol 2005;89:1666-68

11. Goldsberry DH, Ross IB, Dhillon G, et al. Visual dysfunction caused by gauze wrapping of an intracranial aneurysm. J Neuroophthalmol 2004;24:42-45

12. Molyneux AJ, Kerr RS, Stratton I, et al. International Subarachnoid Aneurysm
Trial (ISAT) of neurosurgical clipping versus endovascular coiling in 2143 patients with ruptured intracranial aneurysms: a randomised trial. Lancet 2002;360:1267-74

13. Hoh BL, Carter BS, Budzik RF, et al. Results after surgical and endovascular treatment of paraclinoid aneurysms by a combined neurovascular team. $\mathrm{Neu}$ rosurgery 2001;48:78-89

14. Kallmes DF, Helm GA, Hudson SB, et al. Histologic evaluation of platinum coi embolization in an aneurysm model in rabbits. Radiology 1999;213:217-22

15. Fujiwara NH, Kallmes DF. Healing response in elastase-induced rabbit aneurysms after embolization with a new platinum coil system. AJNR Am J Neuroradiol 2002;23:1137-44

16. Dai D, Ding YH, Kadirvel R. A longitudinal immunohistochemical study of the healing of experimental aneurysms after embolization with platinum coils. AJNR Am J Neuroradiol 2006;27:736-41

17. Dawson RC 3rd, Shengelaia GG, Kisht AF, et al. Histologic effects of collagenfilled interlocking detachable coils in the ablation of experimental aneurysms in swine. AJNR Am J Neuroradiol 1996;17:853-58

18. Desfaits A-C, Raymond J, Muizelaar JP. Growth factors stimulate neointimal cells in vitro and increase the thickness of the neointima formed at the neck of porcine aneurysms treated by embolization. Stroke 2000;31:498-507

19. Graves VB, Strother CM, Rappe AH. Treatment of experimental canine carotid aneurysms with platinum coils. AJNR Am J Neuroradiol 1993;14:787-93

20. Mawad ME, Mawad JK, Cartwright J Jr, et al. Long-term histopathologic changes in canine aneurysms embolized with Guglielmi detachable coils. AJNR Am J Neuroradiol 1995;16:7-13

21. Tamatani S, Ozawa T, Minakawa T, et al. Radiologic and histopathologic evaluation of canine artery occlusion after collagen-coated platinum microcoil delivery. AJNR Am J Neuroradiol 1999;20:541-45

22. Tenjin H, Fushiki S, Nakahara Y, et al. Effect of Guglielmi detachable coils on experimental carotid artery aneurysms in primates. Stroke 1995;26:2075-80

23. Böcher-Schwarz HG, Ringel $\mathrm{K}$, Bohl J, et al. Histological findings in coilpacked experimental aneurysms 3 months after embolization. Neurosurgery 2002;50:379-85

24. Stiver SI, Porter PJ, Willinsky RA, et al. Acute human histopathology of an intracranial aneurysm treated using Guglielmi detachable coils: case report and review of the literature. Neurosurgery 1998;43:1203-08

25. Ishihara S, Mawad ME, Ogata $\mathrm{K}$, et al. Histopathologic findings in human cerebral aneurysms embolized with platinum coils: report of two cases and review of the literature. AJNR Am J Neuroradiol 2002;23:970-74

26. Bavinzski G, Talazoglu V, Killer M, et al. Gross and microscopic histopathological findings in aneurysms of the human brain treated with Guglielmi detachable coils. J Neurosurg 1999;91:284-93

27. Ding YH, Dai D, Lewis DA, et al. Angiographic and histologic analysis of experimental aneurysms embolized with platinum coils, Matrix, and HydroCoil. AJNR Am J Neuroradiol 2005;26:1757-63

28. Im S-H, Han MH, Kwon BJ, et al. Aseptic meningitis after embolization of cerebral aneurysms using hydrogel-coated coils: report of a case. AJNR Am J Neuroradiol 2007;28:511-12

29. Stracke CP, Krings T, Moller-Hartmann W, et al. Severe inflammatory reaction of the optic system after endovascular treatment of a supraophthalmic aneurysm with bioactive coils. AJNR Am J Neuroradiol 2007;28:1401-02

30. Mericle RA, Wakhloo AK, Lopes DK, et al. Delayed aneurysm regrowth and recanalization after Guglielmi detachable coil treatment: case report. J Neurosurg 1998;89:142-45

31. Kwan ESK, Heilman CB, Shucart WA, et al. Enlargement of basilar artery aneurysms following balloon occlusion: "water-hammer effect." J Neurosurg 1991;75:963-68

32. Kawanabe Y, Sadato A, Taki W, et al. Endovascular occlusion of intracranial aneurysms with Guglielmi detachable coils: correlation between coil packing density and coil compaction. Acta Neurochir (Wien) 2001;143:451-55

33. Turjman F, Massoud TF, Sayre J, et al. Predictors of aneurysm occlusion in the period immediately after endovascular treatment with detachable coils: a multivariate analysis. AJNR Am J Neuroradiol 1998;19:1645-51

34. Fernandez Zubillaga AF, Guglielmi G, Vinuela F, et al. Endovascular occlusion of intracranial aneurysms with electrically detachable coils: correlation of aneurysm neck size and treatment results. AJNR Am J Neuroradiol 1994;15:815-20

35. Piotin M, Mandai S, Murphy KJ, et al. Dense packing of cerebral aneurysms: an in vitro study with detachable platinum coils. AJNR Am J Neuroradiol 2000;21:757-60

36. Viñuela F, Duckwiler G, Mawad M. Guglielmi detachable coil embolization of acute intracranial aneurysm: perioperative anatomic and clinical outcome in 403 patients. J Neurosurg 1997;86:475-82

37. Hayakawa M, Murayama Y, Duckwiler GR, et al. Natural history of the neck remnant of a cerebral aneurysm treated with the Guglielmi detachable coil system. J Neurosurg 2000;93:561-68

38. Thornton J, Debrun GM, Aletich VA, et al. Follow-up angiography on intracranial aneurysms treated with endovascular placement of Guglielmi detachable coils. Neurosurgery 2002;50:239-49

39. Bhatti MT, Peters KR, Firment C, et al. Delayed exacerbation of third nerve palsy due to aneurysmal regrowth after endovascular coil embolization. J Neuroophthalmol 2004;24:3-9 